\title{
Ruthenium-mediated Insertion of an Unsaturated C4 Unit into the P-N Bond of an Aminophosphine Ligand
}

\author{
Sonja Pavlik, Florian Jantscher, Kurt Mereiter, and Karl Kirchner*
}

\section{Supporting Information}

General Techniques. All manipulations were performed under an inert atmosphere of argon by using Schlenk techniques. All chemicals were standard reagent grade and used without further purification. The solvents were purified according to standard procedures. ${ }^{1}$ The deuterated solvents were purchased from Aldrich and dried over $4 \AA$ molecular sieves. $\left[\mathrm{RuCp}(\mathrm{MeCN})_{3}\right] \mathrm{PF}_{6}$ was prepared according to the literature. ${ }^{2}{ }^{1} \mathrm{H},{ }^{13} \mathrm{C}\left\{{ }^{1} \mathrm{H}\right\}$, and ${ }^{31} \mathrm{P}\left\{{ }^{1} \mathrm{H}\right\}$ NMR spectra were recorded on a Bruker AVANCE-250 spectrometer operating at $250.13,62.86$, and $101.26 \mathrm{MHz}$, respectively, and were referenced to $\mathrm{SiMe}_{4}$ and $\mathrm{H}_{3} \mathrm{PO}_{4}(85 \%) .{ }^{1} \mathrm{H}$ and ${ }^{13} \mathrm{C}\left\{{ }^{1} \mathrm{H}\right\}$ NMR signal assignments were confirmed by ${ }^{1} \mathrm{H}$-COSY, 135-DEPT, and HSQC $\left({ }^{1} \mathrm{H}-{ }^{13} \mathrm{C}\right)$ experiments.

[RuCp $\left.\left(\mathrm{PPh}_{2} \mathrm{NEt}_{2}\right)\left(\mathrm{CH}_{3} \mathrm{CN}\right)_{2}\right] \mathrm{PF}_{6}$ (1). To a solution of $\left[\mathrm{RuCp}\left(\mathrm{CH}_{3} \mathrm{CN}\right)_{3}\right] \mathrm{PF}_{6}(300 \mathrm{mg}, 0.69$ $\mathrm{mmol})$ in $\mathrm{CH}_{2} \mathrm{Cl}_{2}(5 \mathrm{~mL}) \mathrm{PPh}_{2} \mathrm{NEt}_{2}(179 \mathrm{mg}, 0.69 \mathrm{mmol})$ was added and the mixture was stirred for $2 \mathrm{~h}$ at room temperature. After removal of the solvent, a yellow powder was obtained which was collected on a glass frit, washed with $\mathrm{Et}_{2} \mathrm{O}(3 \times 10 \mathrm{~mL})$, and dried under vacuum. Yield: $416 \mathrm{mg}(93 \%)$. Anal. Calcd. for $\mathrm{C}_{25} \mathrm{H}_{31} \mathrm{~F}_{6} \mathrm{~N}_{3} \mathrm{P}_{2} \mathrm{Ru}$ : C, 46.16; $\mathrm{H}, 4.80 ; \mathrm{N}, 6.46$. Found: $\mathrm{C}, 46.10 ; \mathrm{H}, 4.72 ; \mathrm{N}, 6.55 .{ }^{1} \mathrm{H}$ NMR $(\delta$, $\left.\mathrm{CD}_{2} \mathrm{Cl}_{2}, 20^{\circ} \mathrm{C}\right): 7.61-7.30(\mathrm{~m}, 10 \mathrm{H}, \mathrm{Ph}), 4.26(\mathrm{~s}, 5 \mathrm{H}, \mathrm{Cp}), 3.42-3.20(\mathrm{~m}, 4 \mathrm{H}, \mathrm{Et}), 2.22\left(6 \mathrm{H}, \mathrm{CH}_{3} \mathrm{CN}\right)$, $0.96\left(\mathrm{t}, J_{H H}=7.1 \mathrm{~Hz}, 3 \mathrm{H}, \mathrm{Et}\right) .{ }^{13} \mathrm{C}\left\{{ }^{1} \mathrm{H}\right\} \operatorname{NMR}\left(\delta, \mathrm{CD}_{2} \mathrm{Cl}_{2}, 20^{\circ} \mathrm{C}\right): 137.4\left(\mathrm{~d},{ }^{1} \mathrm{~J}_{C P}=46.0 \mathrm{~Hz}, \mathrm{Ph}^{1}\right), 131.4(\mathrm{~d}$, $\left.{ }^{2} J_{C P}=11.5 \mathrm{~Hz}, \mathrm{Ph}^{2,6}\right), 129.7\left(\mathrm{~d},{ }^{4} J_{C P}=1.9 \mathrm{~Hz}, \mathrm{Ph}^{4}\right), 128.0\left(\mathrm{~d},{ }^{3} J_{C P}=9.6 \mathrm{~Hz}, \mathrm{Ph}^{3,5}\right), 127.3\left(\mathrm{CH}_{3} \mathrm{CN}\right)$, $77.4\left(\mathrm{~d}, J_{C P}=2.3 \mathrm{~Hz}, \mathrm{Cp}\right), 42.4\left(\mathrm{~d}, J_{C P}=6.5 \mathrm{~Hz}, \mathrm{CH}_{2}\right), 13.6\left(\mathrm{~d}, \mathrm{~J}_{C P}=2.3 \mathrm{~Hz}, \mathrm{CH}_{3}\right), 3.7\left(\mathrm{CH}_{3} \mathrm{CN}\right)$. ${ }^{31} \mathrm{P}\left\{{ }^{1} \mathrm{H}\right\} \operatorname{NMR}\left(\delta, \mathrm{CD}_{2} \mathrm{Cl}_{2}, 20^{\circ} \mathrm{C}\right): 102.1\left(\mathrm{PPh}_{2}\right),-143.0\left({ }^{1} \mathrm{~J}_{F P}=711.8 \mathrm{~Hz}, \mathrm{PF}_{6}\right)$.

[RuCp $\left.\left(\eta^{3}-(\mathrm{P}, \mathrm{C}, \mathrm{C})-\mathrm{PPh}_{2} \mathrm{CHC}-\left(\mathrm{CH}_{2}\right)_{3}-\eta^{2}-(\mathrm{C}, \mathrm{C})-\mathrm{CCHNEt}_{2}\right)\right] \mathrm{PF}_{6}(\mathbf{2 a})$. To a solution of 1 (100 mg, $0.15 \mathrm{mmol})$ in $\mathrm{CH}_{2} \mathrm{Cl}_{2}(10 \mathrm{~mL}) 1.1$ equiv of 1,6-heptadiyne (19.5 $\left.\mu \mathrm{L}, 0.17 \mathrm{mmol}\right)$ was added and the mixture was stirred for $2 \mathrm{~h}$ at room temperature. After removal of the solvent under reduced pressure, an orange solid was obtained which was washed with $\mathrm{Et}_{2} \mathrm{O}(5 \mathrm{~mL})$, and dried under vacuum. Yield: 75 mg (52\%). Anal. Calcd. for $\mathrm{C}_{28} \mathrm{H}_{33} \mathrm{~F}_{6} \mathrm{NP}_{2} \mathrm{Ru}$ : C, 50.91; H, 5.09; N, 2.12. Found: $\mathrm{C}, 50.83 ; \mathrm{H}, 5.11 ; \mathrm{N}$, 
2.19. ${ }^{1} \mathrm{H}$ NMR $\left(\delta, \mathrm{CD}_{2} \mathrm{Cl}_{2}, 20^{\circ} \mathrm{C}\right): 7.68-7.41(\mathrm{~m}, 10 \mathrm{H}, \mathrm{Ph}), 5.09(\mathrm{~s}, 5 \mathrm{H}, \mathrm{Cp}), 4.01\left(\mathrm{~d},{ }^{2} \mathrm{~J}_{H P}=4.7 \mathrm{~Hz}\right.$, $\left.1 \mathrm{H}, \mathrm{H}^{1}\right), 3.58\left(\mathrm{~d}, J_{H P}=5.7 \mathrm{~Hz}, 1 \mathrm{H}, \mathrm{H}^{4}\right), 3.01-2.86(\mathrm{~m}, 2 \mathrm{H}), 2.82-2.51(\mathrm{~m}, 6 \mathrm{H}), 2.39-2.27(\mathrm{~m}, 2 \mathrm{H})$, $0.96\left(\mathrm{t}, J_{H H}=7.1 \mathrm{~Hz}, 3 \mathrm{H}\right) .{ }^{13} \mathrm{C}\left\{{ }^{1} \mathrm{H}\right\} \operatorname{NMR}\left(\delta, \mathrm{CD}_{2} \mathrm{Cl}_{2}, 20^{\circ} \mathrm{C}\right): 133.2\left(\mathrm{~d},{ }^{2} J_{C P}=11.9 \mathrm{~Hz}, \mathrm{Ph}^{2,6}\right), 132.4(\mathrm{~d}$, $\left.{ }^{2} J_{C P}=12.3 \mathrm{~Hz}, \mathrm{Ph}^{2^{\prime}, 6^{\prime}}\right), 132.1\left(\mathrm{~d},{ }^{4} J_{C P}=3.5 \mathrm{~Hz}, \mathrm{Ph}^{4}\right), 131.6\left(\mathrm{~d},{ }^{4} J_{C P}=2.3 \mathrm{~Hz}, \mathrm{Ph}^{4}\right), 129.8\left(\mathrm{~d},{ }^{3} J_{C P}=\right.$ $\left.11.5 \mathrm{~Hz}, \mathrm{Ph}^{3,5}\right), 129.0\left(\mathrm{~d},{ }^{3} \mathrm{~J}_{C P}=11.9 \mathrm{~Hz}, \mathrm{Ph}^{3^{\prime}, 5^{\prime}}\right), 125.9\left(\mathrm{~d},{ }^{1} J_{C P}=47.9 \mathrm{~Hz}, \mathrm{Ph}^{1}\right), 117.8\left(\mathrm{~d}, J_{C P}=6.9 \mathrm{~Hz}\right.$, $\left.C^{4}\right), 117.4\left(\mathrm{~d}, J_{C P}=4.6 \mathrm{~Hz}, \mathrm{C}^{3}\right), 79.1\left(\mathrm{~d}, J_{C P}=1.5 \mathrm{~Hz}, \mathrm{Cp}\right), 75.8\left(\mathrm{C}^{2}\right), 46.8\left(\mathrm{CH}_{2}\right), 40.8\left(\mathrm{~d}, J_{C P}=24.2\right.$ $\left.\mathrm{Hz}, \mathrm{C}^{1}\right), 36.0\left(\mathrm{~d}, J_{C P}=7.3 \mathrm{~Hz}, \mathrm{CH}_{2}\right), 33.2\left(\mathrm{CH}_{2}\right), 23.1\left(\mathrm{CH}_{2}\right), 12.1\left(\mathrm{CH}_{3}\right) .{ }^{31} \mathrm{P}\left\{{ }^{1} \mathrm{H}\right\} \operatorname{NMR}\left(\delta, \mathrm{CD}_{2} \mathrm{Cl}_{2}\right.$, $\left.20^{\circ} \mathrm{C}\right): 4.2\left(\mathrm{PPh}_{2}\right),-143.0\left({ }^{1} J_{F P}=710.8 \mathrm{~Hz}, \mathrm{PF}_{6}\right)$.

Reaction of 1 with 1,7-octadiyne in $\mathrm{CD}_{2} \mathrm{Cl}_{2}$. Formation of $\left[\mathrm{RuCp}\left(\eta^{3}-\left(\mathrm{P}, \mathrm{C}, \mathrm{C}-\mathrm{PPh}_{2}-\mathrm{CH}-\right.\right.\right.$ $\left.\mathrm{C}\left(\mathrm{CH}_{2}\right)_{4}-\eta^{2}-(\mathrm{C}, \mathrm{C})-\mathrm{CCHNEt}_{2}\right] \mathrm{PF}_{6}(2 \mathrm{~b})$ and $\left[\mathrm{RuCp}\left(\eta^{1}-(\mathrm{P})-\mathrm{PPh}_{2}-\mathrm{CH}=\mathrm{C}\left(\mathrm{CH}_{2}\right)_{4}-\eta^{3}-(\mathrm{C}, \mathrm{C}, \mathrm{N})-\mathrm{CCHNEt}_{2}\right] \mathrm{PF}_{6}\right.$ (3b). A $5 \mathrm{~mm}$ NMR tube was charged with 1 (30 mg, $0.05 \mathrm{mmol})$ in $\mathrm{CD}_{2} \mathrm{Cl}_{2}(0.5 \mathrm{~mL})$ and 1,7-octadiyne $(6,1 \mu \mathrm{L}, 0.05 \mathrm{mmol})$ was added via syringe. The reaction was then monitored by ${ }^{1} \mathrm{H}$ and ${ }^{31} \mathrm{P}\left\{{ }^{1} \mathrm{H}\right\} \mathrm{NMR}$ spectroscopy. After $20 \mathrm{~min}$ both $\mathbf{2 b}$ and $\mathbf{3 b}$ are formed in an approximately $3: 1$ ratio. After $5 \mathrm{~h}$ this ratio already changed to ca. 1:6. Due to spectral overlap with $\mathbf{3 b}$ only the most characteristic signals of $\mathbf{2 b}$ could be unequivocally assigned. ${ }^{1} \mathrm{H} \operatorname{NMR}\left(\delta, \mathrm{CD}_{2} \mathrm{Cl}_{2}, 20^{\circ} \mathrm{C}\right): 5.10(\mathrm{~s}, 5 \mathrm{H}, \mathrm{Cp}), 3.53\left(\mathrm{~d},{ }^{2} J_{H P}=4.7 \mathrm{~Hz}\right.$, $\left.1 \mathrm{H}, \mathrm{H}^{1}\right), 2.90\left(\mathrm{~d}, J_{H P}=7.6 \mathrm{~Hz}, 1 \mathrm{H}, \mathrm{H}^{4}\right), 0.83\left(\mathrm{t}, J_{H H}=7.1 \mathrm{~Hz}, 6 \mathrm{H}, \mathrm{Et}\right) .{ }^{31} \mathrm{P}\left\{{ }^{1} \mathrm{H}\right\} \mathrm{NMR}\left(\delta, \mathrm{CD}_{2} \mathrm{Cl}_{2}, 20^{\circ} \mathrm{C}\right)$ : 3.3 $\left(\mathrm{PPh}_{2}\right),-143.0\left({ }^{1} \mathrm{~J}_{F P}=710.8 \mathrm{~Hz}, \mathrm{PF}_{6}\right)$. After heating at $40^{\circ} \mathrm{C}$ for $8 \mathrm{~h}, \mathbf{2 b}$ is completely converted to 3b.

$\left[\mathrm{RuCp}\left(\eta^{1}-(\mathrm{P})-\mathrm{PPh}_{2} \mathrm{CH}=\mathrm{C}-\left(\mathrm{CH}_{2}\right)_{3}-\eta^{3}-(\mathrm{C}, \mathrm{C}, \mathrm{N})-\mathrm{CCHNEt}_{2}\right)\right] \mathrm{PF}_{6}$ (3a). A stirred solution of $2 \mathrm{a}(50$ $\mathrm{mg}, 0,08 \mathrm{mmol})$ in $\mathrm{CH}_{3} \mathrm{NO}_{2}(5 \mathrm{~mL})$ was kept overnight at $80^{\circ} \mathrm{C}$. After removal of the solvent under reduced pressure, an orange solid was obtained which was washed with $\mathrm{Et}_{2} \mathrm{O}(5 \mathrm{~mL})$, and dried under vacuum. Yield: $45 \mathrm{mg}(85 \%)$. Anal. Calcd. for $\mathrm{C}_{28} \mathrm{H}_{33} \mathrm{~F}_{6} \mathrm{NP}_{2} \mathrm{Ru}$ : C, 50.91; H, 5.04; N, 2.12. Found: C, 50.87; $\mathrm{H}, 5.11 ; \mathrm{N}, 2.08 .{ }^{1} \mathrm{H}$ NMR $\left(\delta, \mathrm{CD}_{3} \mathrm{NO}_{2}, 20^{\circ} \mathrm{C}\right): 8.08-7.88(\mathrm{~m}, 2 \mathrm{H}, \mathrm{Ph}), 7.73-7.34(\mathrm{~m}, 6 \mathrm{H}, \mathrm{Ph})$, $7.33-7.16(\mathrm{~m}, 2 \mathrm{H}, \mathrm{Ph}), 6.35\left(\mathrm{~d}, J_{H P}=9.8 \mathrm{~Hz}, 1 \mathrm{H}, \mathrm{H}^{1}\right), 6.03\left(\mathrm{~s}, 1 \mathrm{H}, \mathrm{H}^{4}\right), 4.76(\mathrm{~s}, 5 \mathrm{H}, \mathrm{Cp}), 3.72-3.51$ (m, 1H), $3.03-2.44(\mathrm{~m}, 4 \mathrm{H}), 2.27-1.84(\mathrm{~m}, 5 \mathrm{H}), 1.21\left(\mathrm{t}, J_{H H}=7.1 \mathrm{~Hz}, 3 \mathrm{H}\right), 0.81\left(\mathrm{t}, J_{H H}=7.1 \mathrm{~Hz}, 3 \mathrm{H}\right)$. ${ }^{13} \mathrm{C}\left\{{ }^{1} \mathrm{H}\right\} \operatorname{NMR}\left(\delta, \mathrm{CD}_{3} \mathrm{NO}_{2}, 20^{\circ} \mathrm{C}\right): 172.0\left(\mathrm{~d}, J_{C P}=27.2 \mathrm{~Hz}, \mathrm{C}^{3}\right), 137.5\left(\mathrm{~d},{ }^{1} J_{C P}=54.4 \mathrm{~Hz}, \mathrm{Ph}^{1}\right), 134.0(\mathrm{~d}$, $\left.{ }^{2} J_{C P}=12.3 \mathrm{~Hz}, \mathrm{Ph}^{2,6}\right), 131.6\left(\mathrm{~d},{ }^{1} J_{C P}=47.2 \mathrm{~Hz}, \mathrm{Ph}^{1}\right), 131.3\left(\mathrm{~d},{ }^{4} J_{C P}=2.3 \mathrm{~Hz}, \mathrm{Ph}^{4}\right), 130.4\left(\mathrm{~d},{ }^{2} J_{C P}=\right.$ $\left.10.7 \mathrm{~Hz}, \mathrm{Ph}^{2^{\prime}, 6^{\prime}}\right), 129.9\left(\mathrm{~d},{ }^{4} J_{C P}=2.3 \mathrm{~Hz}, \mathrm{Ph}^{4}\right), 128.7\left(\mathrm{~d},{ }^{3} J_{C P}=10.0 \mathrm{~Hz}, \mathrm{Ph}^{3,5}\right), 128.6\left(\mathrm{~d},{ }^{3} J_{C P}=10.7\right.$ $\left.\mathrm{Hz}, \mathrm{Ph}^{3^{3}, 5^{\prime}}\right), 117.4\left(\mathrm{~d}, J_{C P}=47.9 \mathrm{~Hz}, \mathrm{C}^{1}\right), 91.3\left(\mathrm{~d}, J_{C P}=3.1 \mathrm{~Hz}, \mathrm{C}^{2}\right), 82.2\left(\mathrm{~d}, J_{C P}=1.9 \mathrm{~Hz}, \mathrm{Cp}\right), 79.1(\mathrm{~d}$, $\left.J_{C P}=1.5 \mathrm{~Hz}, \mathrm{C}^{4}\right), 58.3\left(\mathrm{CH}_{2}\right), 44.1\left(\mathrm{~d}, J_{C P}=8.8 \mathrm{~Hz}, \mathrm{CH}_{2}\right), 38.4\left(\mathrm{CH}_{2}\right), 28.8\left(\mathrm{~d}, J_{C P}=19.6 \mathrm{~Hz}, \mathrm{CH}_{2}\right)$, 
$25.1\left(\mathrm{CH}_{2}\right), 13.8\left(\mathrm{CH}_{3}\right), 12.1\left(\mathrm{CH}_{3}\right) \cdot{ }^{31} \mathrm{P}\left\{{ }^{1} \mathrm{H}\right\} \operatorname{NMR}\left(\delta, \mathrm{CD}_{2} \mathrm{Cl}_{2}, 20^{\circ} \mathrm{C}\right): 74.1\left(\mathrm{PPh}_{2}\right),-143.3\left({ }^{1} \mathrm{~J}_{F P}=707.1\right.$ $\left.\mathrm{Hz}, \mathrm{PF}_{6}\right)$.

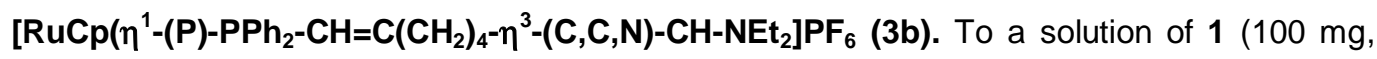
$0.15 \mathrm{mmol})$ in $\mathrm{CH}_{2} \mathrm{Cl}_{2}(10 \mathrm{~mL}) 1.1$ equiv of 1,7 -octadiyne $(20.8 \mu \mathrm{L}, 0.15 \mathrm{mmol})$ was added and the mixture was heated for $8 \mathrm{~h}$ at $40^{\circ} \mathrm{C}$. After removal of the solvent under reduced pressure, an orange solid was obtained which was washed with $\mathrm{Et}_{2} \mathrm{O}(5 \mathrm{~mL})$, and dried under vacuum. Yield: $56 \mathrm{mg}(55 \%)$. Anal. Calcd. for $\mathrm{C}_{29} \mathrm{H}_{35} \mathrm{~F}_{6} \mathrm{NP}_{2} \mathrm{Ru}$ : C, 51.63; $\mathrm{H}, 5.23 ; \mathrm{N}, 2.08$. Found: $\mathrm{C}, 51.55 ; \mathrm{H}, 5.27 ; \mathrm{N}, 2.17 .{ }^{1} \mathrm{H}$ NMR $\left(\delta, \mathrm{CD}_{2} \mathrm{Cl}_{2}, 20^{\circ} \mathrm{C}\right): 7.96-7.79(\mathrm{~m}, 2 \mathrm{H}, \mathrm{Ph}), 7.66-7.39(\mathrm{~m}, 6 \mathrm{H}, \mathrm{Ph}), 7.34-7.18(\mathrm{~m}, 2 \mathrm{H}, \mathrm{Ph}), 6.21(\mathrm{~d}$, $\left.J_{H P}=9.8 \mathrm{~Hz}, 1 \mathrm{H}, \mathrm{H}^{1}\right), 6.06\left(\mathrm{~s}, 1 \mathrm{H}, \mathrm{H}^{4}\right), 4.69(\mathrm{~s}, 5 \mathrm{H}, \mathrm{Cp}), 3.78-3.57(\mathrm{~m}, 1 \mathrm{H}), 3.28-3.12(\mathrm{~m}, 1 \mathrm{H}), 2.72$ $-2.40(\mathrm{~m}, 2 \mathrm{H}), 2.34-1.68(\mathrm{~m}, 8 \mathrm{H}), 1.22\left(\mathrm{t}, J_{H H}=7.0 \mathrm{~Hz}, 3 \mathrm{H}\right), 0.89\left(\mathrm{t}, J_{H H}=7.1 \mathrm{~Hz}, 3 \mathrm{H}\right) .{ }^{13} \mathrm{C}\left[{ }^{1} \mathrm{H}\right\} \mathrm{NMR}$ $\left(\delta, \mathrm{CD}_{2} \mathrm{Cl}_{2}, 20^{\circ} \mathrm{C}\right): 169.5\left(\mathrm{~d}, J_{C P}=25.3 \mathrm{~Hz}, \mathrm{C}^{3}\right), 137.8\left(\mathrm{~d},{ }^{1} J_{C P}=54.1 \mathrm{~Hz}, \mathrm{Ph}^{1}\right), 134.1\left(\mathrm{~d},{ }^{2} J_{C P}=12.3\right.$ $\left.\mathrm{Hz}, \mathrm{Ph}^{2,6}\right), 131.8\left(\mathrm{~d},{ }^{4} J_{C P}=2.3 \mathrm{~Hz}, \mathrm{Ph}^{4}\right), 131.1\left(\mathrm{~d},{ }^{1} J_{C P}=45.6 \mathrm{~Hz}, \mathrm{Ph}^{1}\right), 130.3\left(\mathrm{~d},{ }^{4} \mathrm{~J}_{C P}=2.3 \mathrm{~Hz}, \mathrm{Ph}^{4}\right)$, $130.3\left(\mathrm{~d},{ }^{2} J_{C P}=10.7 \mathrm{~Hz}, \mathrm{Ph}^{2}, 6^{\prime}\right), 129.0\left(\mathrm{~d},{ }^{3} J_{C P}=10.3 \mathrm{~Hz}, \mathrm{Ph}^{3,5}\right), 122.5\left(\mathrm{~d}, J_{C P}=48.0 \mathrm{~Hz}, \mathrm{C}^{1}\right), 90.8(\mathrm{~d}$, $\left.J_{C P}=2.7 \mathrm{~Hz}, \mathrm{C}^{2}\right), 82.7\left(\mathrm{~d}, J_{C P}=1.9 \mathrm{~Hz}, \mathrm{Cp}\right), 79.8\left(\mathrm{C}^{4}\right), 58.4\left(\mathrm{CH}_{2}\right), 44.9\left(\mathrm{CH}_{2}\right), 43.8\left(\mathrm{~d}, J_{C P}=6.5 \mathrm{~Hz}\right.$, $\left.\mathrm{CH}_{2}\right), 36.2\left(\mathrm{~d}, J_{C P}=21.5 \mathrm{~Hz}, \mathrm{CH}_{2}\right), 29.9\left(\mathrm{CH}_{2}\right), 28.3\left(\mathrm{CH}_{2}\right), 14.2\left(\mathrm{CH}_{3}\right), 12.9\left(\mathrm{CH}_{3}\right) .{ }^{31} \mathrm{P}\left\{{ }^{1} \mathrm{H}\right\} \operatorname{NMR}(\delta$, $\left.\mathrm{CD}_{2} \mathrm{Cl}_{2}, 20^{\circ} \mathrm{C}\right): 76.3\left(\mathrm{PPh}_{2}\right),-142.9\left({ }^{1} J_{F P}=710.8 \mathrm{~Hz}, \mathrm{PF}_{6}\right)$.

1 Perrin, D. D.; Armarego, W. L. F.Purification of Laboratory Chemicals, 3rd ed.; Pergamon: New York, 1988.

2 Gill; T. P.; Mann, K. R. Organometallics 1982, 1, 485. 